
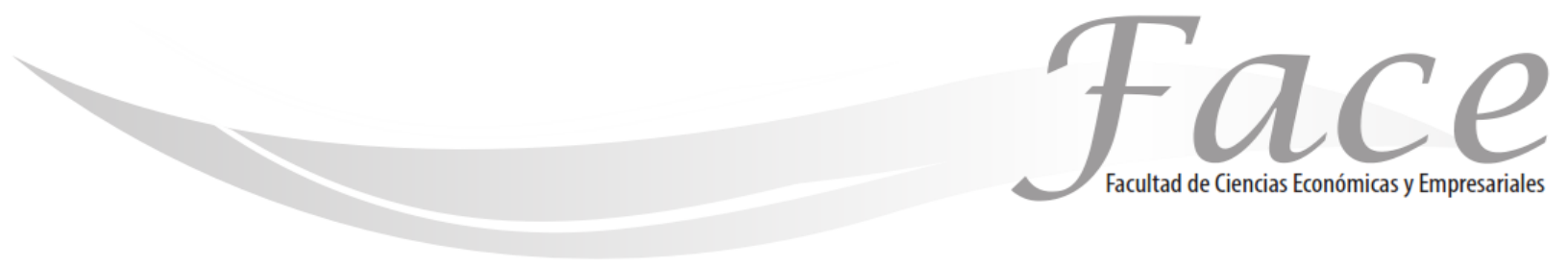

ISSN Impreso: 1794-9920

ISSN Electrónico: 2500-9338

Volumen $19-\mathrm{N}^{\circ} 1$

Año 2019

Págs. $39-49$

\title{
POLÍTICA PÚBLICA SOBRE MIGRACIÓN DE RETORNO. UN DESAFÍO Y OPORTUNIDAD DE MERCADO PARA UNIVERSIDADES
}

\author{
Blanca Isabel Llamas Félix * \\ ORCID: https://orcid.org/0000-0002-0782-8340 \\ Víctor Hugo Bañuelos García* \\ ORCID: https://orcid.org/0000-0003-0888-4157 \\ Flor de María García Martínez* \\ ORCID: https://orcid.org/0000-0003-3869-0169
}

Fecha de Recepción: Marzo 5 de 2019

Fecha de Aprobación: Junio 12 de 2019

\section{Resumen:}

El presente trabajo estudia las políticas públicas en torno a la migración México-Estados Unidos, hace un breve recorrido por los antecedentes, desarrollo y condiciones actuales sobre dicho desafío, el perfil de quienes constituyen el migrante de retorno, sus características y aptitudes; para determinar los requerimientos de capacitación o continuidad de estudios y, una vez analizada dicha información, estar en condiciones de diseñar estrategias de mercado, por estudiantes de nivel licenciatura para su inserción al sistema educativo, particularmente universidades de ser el caso, y así proveerles de las herramientas necesarias para incorporarlos al mercado laboral, puesto que México tiene gran flujo migratorio a su vecino país del norte, del cual en los últimos años está siendo víctima de fuertes deportaciones masivas.

Palabras clave: Políticas públicas, Migrante de retorno, Estrategia de mercado

*Docente Investigadora Tiempo Completo Universidad Autónoma de Zacatecas - México, Contacto: blancaisabel@unizacatecas.edu.mx

** Docente Investigador Tiempo Completo Universidad Autónoma de Zacatecas - México, .Contacto: bag_70@hotmail.com

*** Docente Investigadora Tiempo Completo Universidad Autónoma de Zacatecas - México, Contacto: florecitagama@hotmail.com 


\title{
PUBLIC POLICY ON RETURN MIGRATION. A CHALLENGE AND MARKET OPPORTUNITY FOR UNIVERSITIES
}

\begin{abstract}
:
The present paper is made about the public policies surrounding migration between Mexico-United States of America, makes a brief tour through the antecedents of said challenge, the profile of those who make up the returning migrant, their characteristics and aptitudes; to determine the requirements of training or the continuity of studies and once this information is analyzed, being in condition to design market strategies, by degree students, for their insertion in the educational system, particularly universities, that being the case, and so on providing the necessary tools to incorporate them to the working market, this due to Mexico having a great migratory flow to its neighbor country of the north, of which it has being suffering greater, massive deportations.
\end{abstract}

Keywords: Public policies, returning migrant, market strategies

\section{DIMENSÕES DO TRABALHO DA SÍNDROME DE BURNOUT NO PROFESSOR-PESQUISADOR DE UNIVERSIDADES PÚBLICAS}

\begin{abstract}
Resumo:
O presente trabalho é feito sobre as políticas públicas em torno da migração entre México-Estados Unidos da América, faz um breve passeio pelos antecedentes do referido desafio, o perfil daqueles que compõem o migrante retornando, suas características e aptidões; determinar as exigências de formação ou a continuidade de estudos e uma vez que esta informação é analisada, estando em condições de desenhar estratégias de mercado, por estudantes de graduação, para sua inserção no sistema educacional, particularmente universidades, sendo assim, e assim por diante. as ferramentas necessárias para incorporá-las ao mercado de trabalho, isso devido ao fato de o México ter um grande fluxo migratório para o país vizinho do norte, do qual está sofrendo deportações maiores e em massa.
\end{abstract}

Palavras-chave: Políticas públicas, retorno de migrantes, estratégias de mercado 


\section{INTRODUCCIÓN:}

En el marco de la actual crisis de movilidad existente en el mundo, es importante responder la pregunta acerca del desafío y a la vez oportunidad, que representa el vulnerable grupo migrante de retorno para las políticas públicas y los mercados existentes, así como la inminente necesidad de que las instituciones educativas, planteen estrategias de mercado que faciliten su inserción a la sociedad y al mercado laboral en su nuevo entorno.

Plantear políticas públicas en materia educativa, respecto de migración y desarrollo no pretende sugerir ingenuamente que el Estado mexicano las adoptará como suyas, más bien se refiere a un proceso social que se nutre y reclama a la vez de la participación de múltiples actores educativos, políticos, sociales y económicos interesados y comprometidos con el desarrollo del país (Delgado, 2016).

La migración mexicana con destino a los Estados Unidos tiene su origen en 1837, fenómeno agravado en los siglos XIX y XX, como consecuencia de la guerra, donde le arrebataron parte del territorio nacional, provocando que quienes fueran despojados de sus tierras, migraran, aunque el flujo migratorio realmente fuerte, se relaciona con el mercado de trabajo generado por la agricultura (Durand J. y., 2003).

La migración se incrementó durante la Revolución Maderista y a la conclusión de un régimen autoritario que duró más de 30 años (Mercado Vargas, 2006).

Hasta mediados de los ochenta el migrante mexicano procedía de las localidades rurales del país, sin embargo, en los últimos años, quienes eligen como destino Estados Unidos, se ha visto incrementado por mujeres sin la compañía de varón, procedentes de ciudades, de nuevas regiones y hasta con preparación universitaria.

México a lo largo de la historia ha sido exportador de mano de obra hacia Estados Unidos, desde el siglo pasado en los años setenta y continúa hasta la fecha, sin embargo, en los últimos años se ha registrado un considerable aumento en el retorno de ellos.

La migración México-Estados Unidos crece a ritmos acelerados y experimenta significativas transformaciones que dan cuerpo a una dialéctica particular. Se generan dinámicas regresivas (pérdida de trabajadores calificados, progresiva dependencia de las remesas, desarticulación y estancamiento productivo, inflación, desintegración familiar, profundización de desigualdades sociales, etc.) que convergen, progresivamente, en una creciente y preocupante tendencia al despoblamiento y abandono de actividades productivas en las zonas de fuerte migración ( (Delgado, 2016).

La migración tiene múltiples características, entre las negativas está la separación de familias, dispersión de sus integrantes y hasta los problemas de comunicación por el no dominio de la lengua cuando migran, como cuando retornan, mientas que entre las positivas está la mejor calidad de vida de quien lo hace y de sus familias, así como el envío de remesas de dinero.

En 2000 el retorno de migrantes era un flujo formado por trabajadores jubilados y por aquellos que decidieron no mantenerse en la migración, para 2013, fueron los migrantes ya establecidos y en plena edad productiva, mientras que en los últimos años, es por la política antiinmigrante propuesta por los mandatarios Barack Obama y Donald Trump, que hicieron al migrante, objeto de deportación (Moctezuma L., 2013).

(Moctezuma L., 2013), en análisis sobre indicadores de la CONAPO acerca del Índice de Intensidad Migratoria, observan que en 2010 el porcentaje de hogares y/o viviendas con migrantes en Estados Unidos se redujo, mientras que el de viviendas con migrantes de retorno aumentó, por tal, dicha tendencia indica que entre 2005 y 2010 se produjo una reducción drástica de la emigración mexicana hacia Estados Unidos, donde una parte de los establecidos se transformó en migrante de retorno.

Las políticas de deportación se ven acentuadas por el problema de discriminación, encontrando que las de los inmigrantes mexicanos se realizaron en la década de los treinta del siglo XX, aunque muchos de los datos de que se tiene conocimiento incluyen a la población latina o hispana en general, que según United States Census Bureau, 2010, del total de la población latina o hispana censada en Estados Unidos, 63\% era de origen mexicano (Soria, 2017).

Investigaciones sobre discriminación de migrantes mexicanos reportan que, (Ayers. J. W., 2009) 31\% de los latinos, conoce casos de discriminación por causa del idioma y la apariencia física, señalando que los prejuicios raciales de los "anglos" determinan las actitudes en contra de la migración, que están determinadas por el resentimiento racial y que desembocan en deportaciones masivas.

El actual fenómeno migratorio presenta retos considerables que demandan la generación y diseño de estrategias de mercado para atender dicha realidad, que además contribuya al desarrollo económico y social del país. 
El migrante de retorno se convierte en un gran desafío para el mercado, principalmente por la falta de empleos, así que es necesario establecer estrategias de mercado que le permitan su reincorporación al mercado laboral, con la correspondiente observancia de los derechos humanos, a fin de reducir los riesgos a los que éstos se enfrentan en México.

Los recientes cambios observados en la migración, se convierten en grandes e importantes retos para los países destino, pero más aún para los países origen, ya que en el caso de México, no sólo enfrenta la problemática que representa la enorme cantidad de mexicanos en Estados Unidos, sino el creciente flujo de retorno de connacionales, de centroamericanos, más los que solicitan refugio y asilo político.

En México algunos de los migrantes llegan sin empleo, pero con ciertas habilidades que adquirieron a lo largo de los años en el extranjero, de un posible ahorro de recursos económicos para emprender un negocio e incluso de las remesas enviadas a sus familiares en el país, siendo justo en este caso donde se presenta el reto para las instituciones educativas y los estudiantes que las conforman, a fin de generar estrategias de mercado para su reinserción.

Expertos en la materia, afirman que los migrantes que poseen mayor cúmulo de habilidades son los que han permanecido por mayor tiempo en Estados Unidos, es decir, los que logran tener pleno dominio del inglés y una preparación académica aceptable. En ese mismo tenor (Autler, 1997), "la transferencia de destrezas entre los retornados fue más frecuente mientras mayor fue el tiempo de permanencia y más alto el nivel de estatus laboral en el extranjero..."

Es importante el tipo de habilidades y preparación adquiridas, pues tienen que ver con el contexto socioeconómico en que se insertan, ya que en ocasiones, el entorno no ofrece las condiciones adecuadas para desarrollarlas, surgiendo la conveniencia de partir de aquí para que los estudiantes propicien el aprovechamiento de dichas destrezas y los acompañen durante inserción al mercado laboral.

Se pretende entender el contexto de retorno y en éste el rol del retornado, para diseñar estrategias de mercado para su reinserción, por tal se analiza la experiencia de retorno, no necesariamente definitiva y permanente, pero si lo suficiente para que vía estudios de mercado se determine el ámbito donde desarrollarán al máximo su potencial.

Los hombres y mujeres mexicanas que han llegado a México deportadas, en la actualidad debieran tener un lugar importante en la economía del país, con el interés de que vayan emprendiendo negocios, pues significan una forma de participación activa dentro de la sociedad, lo que representa un ingreso, ocupación de mano de obra y una forma de salir de la pobreza, entre otros aspectos y permitiendo así, la participación activa de los estudiantes universitarios.

(Canales, 2012) "en diversos textos se señala que como efecto de la crisis económica actual, la tendencia de crecimiento se habría frenado casi por completo, producto principalmente de un sustancial incremento de la migración de retorno. La pérdida de empleos y oportunidades económicas en los Estados Unidos, junto a la consolidación de políticas restrictivas y discriminatorias en materia de migración, habrían derivado en un freno de la emigración, y un impulso del retorno de una alta proporción de los mexicanos residentes en ese país".

Pese a lo anterior, con base en las estadísticas publicadas por los diferentes organismos nacionales e internacionales, el retorno de los migrantes no ha sido masivo, como se especuló ante las políticas públicas establecidas por la administración actual de Estados Unidos. No obstante, la migración sigue siendo constante. Es importante reconocer que la población migrante jubilada vuelve a su país, en cuyo caso, no existe un interés palpable de incorporarse a la vida laboral o productiva en México.

Es pues de importancia relevante el presente trabajo, para que a partir de obtener un diagnóstico sobre el estado que guarda la migración de retorno, los estudiantes universitarios estén en condiciones de desarrollar estrategias de mercado para dicho segmento, con el fin de direccionar o reorientar los programas sociales que están autorizados, con recursos asignados a dicho sector vulnerable para que puedan iniciar como empresarios, obtener un empleo que les permita salir adelante de sus problemas económicos y elevar su calidad de vida.

\section{MARCO TEÓRICO:}

La situación actual de las políticas públicas en México frente a la migración, parte desde que México no renovó más los programas de braceros, existentes entre 1942 y 1964, misma que se registraba de manera relativamente libre, es decir, con baja intervención del gobierno, beneficiando tanto a empleadores como a trabajadores. 
La promulgación de La Ley sobre Reforma y Control de la Inmigración (IRCA), en 1986, obligó al gobierno mexicano a reflexionar sobre sus posiciones y actitudes, aunque continuaron basándose en el supuesto de que era inevitable, por lo que no se intentó diseñar políticas públicas para revertir sus condiciones económicas y sociales (Alba, Las policas públicas ante el estado de la migración, 2016).

Se esperaba que el TLC generara más trabajos y mejores salarios, lo que podría reducir las presiones migratorias, pero no ocurrió así, por lo que hubiera sido necesario adoptar políticas públicas para desincentivarla, cosa que no ocurrió.

La política seguida por el país fue proteger a los migrantes, reforzando las funciones consulares, mientras que en los noventas, tendientes a acentuar el valor de las remesas y su contribución a la economía del país.

En el 2001, se inició un proceso para el flujo ordenado de trabajadores temporales y permanentes, lo que arrojó resultados similares a los anteriores, aunque más allá de lo que ya ha pasado, el gobierno actual parece no contar con iniciativas importantes, ni para frenarla, ni para enfrentarla, peor aún, ante la enorme carencia de empleo para retener a la población dentro del país (Alba, 1999).

En la actualidad, no obstante el gobierno actual pareciera no contar con iniciativas importantes, la situación de la migración se mueve en dos vertientes: se ha avanzado en el ordenamiento de la migración en la zona fronteriza del sur, se promueve la creación de empleos y la búsqueda de condiciones para incentivar y promover la inversión privada, para retener a la población en el país, de igual manera, el establecimiento de programas de trabajadores temporales predomina, tal como sucede a nivel internacional.

No obstante lo anterior, se evidencia la urgente necesidad de generar políticas públicas, que den salida a los problemas que enfrentan los connacionales una vez que retornan al país, puesto que se enfrentan a la realidad de la cual habían salido huyendo, pero que desafortunadamente no ha presentado cambio alguno.

El fenómeno migratorio ha sido analizado desde hace más de un siglo, aunque en los últimos años ha cobrado gran importancia, en la medida en que la intensidad de los flujos ha aumentado a nivel mundial. Cifras de la División de Población de la ONU indican que el número de inmigrantes internacionales en el mundo aumenta a pasos agigantados, México no es la excepción, por tal, la presente aborda dicha problemática y la propuesta de Mercadotecnia para enfrentarla.

La migración es el cambio de residencia de una o varias personas de manera temporal o definitiva, generalmente con la intención de mejorar su situación económica, su desarrollo personal y familiar. Cuando una persona deja el municipio, estado o país donde reside para irse a vivir a otro lugar se convierte en un emigrante, pero al llegar a establecerse a uno nuevo, pasa a ser un inmigrante (INEGI, 2016).

Las personas migrantes son sujetos integrantes de un colectivo particularmente vulnerable, como sujetos titulares de derechos humanos, destinatarios de determinadas políticas públicas, que son formuladas en torno a la condición migrante de los sujetos a que se dirigen (Gómez, 2014).

Se exige de los poderes públicos, una mayor posición de garante y promotor de los derechos fundamentales del migrante, orientando la acción de promoción de las políticas públicas para abordar el fenómeno migratorio (Gómez, 2014).

De acuerdo con la ONU "el migrante internacional es cualquier persona que cambia su país de residencia usual, es decir, el país donde vive". Por otro lado, George Bojas define a la migración internacional como "el movimiento de las personas o familias a través de las fronteras nacionales" (Durand J. , 2006).

La segmentación del mercado de trabajo es parte del proceso migratorio. En un principio la migración fue para los países destino una oportunidad de tener mano de obra barata, sin embargo, en la actualidad han dejado de ser bienvenidos, no se diga aquellos con deficiente capacitación, ya que no aportan al crecimiento ni desarrollo para los países destino.

Cuando las personas llegan de los países pobres a los ricos, sin conocimiento de la localidad o nexos, no están familiarizadas con las formas de trabajo local, entonces su puesto es en un nivel bajo, la cuestión es si más adelante tendrán una oportunidad para la 
movilidad ascendente, respuesta que depende de las políticas de Estado (Castles \& Miller, 2004).

Aunado al fenómeno migratorio se encuentra el de retorno, del cual poco se ha dicho y por tal estudiado para permitir entenderle, así como explicar una realidad compleja y cambiante.

Hoy en día se está ante un retorno de migrantes que abarca i) repatriaciones, ii) retornos voluntarios individuales y iii) retornos de tipo familiar, al igual que la drástica reducción de emigración (Moctezuma L., 2013), que acertadamente argumenta el autor, es un error suponer que los migrantes regresan al país porque se abrieron nuevas oportunidades.

Lo anterior no debiera ser un problema para el migrante, siempre y cuando las instituciones de educación superior se involucraran en dicha problemática y pusieran al servicio de la sociedad, estudiantes que vía estudios de mercado, planes de negocios, campañas promocionales, entre otras, ofrecieran a dicho segmento de mercado, soluciones a la situación en que se encuentran al regresar a sus países, mismos que parecen no proporcionarles oportunidad alguna.

Existen tres tipos de migrantes: el migrante ahorrador, que envía pequeñas pero constantes remesas a sus familiares para realizar inversiones, adquisición de casas y terrenos, entre otras; el migrante empresario, que es aquel que ha pasado de una persona que ahorra a otra que invierte en empresas o crea empresas, con la intención de generar utilidades en la compra-venta de bienes y servicios; y por último el migrante retirado de la carrera migratoria, que es aquel que posee ciertas habilidades que pueden contribuir a la economía de su país, una vez que ha retornado al mismo (Moctezuma Longoria, 2011).

Para lograr lo anterior, será necesario que los ahorros de los migrantes se puedan invertir en negocios, pero no sólo eso, sino que demuestren que poseen habilidades para trabajarlos con conocimientos previos orientados a cómo contribuir a sus finanzas personales y a su vez, a la economía del país al cual retornan; por tal se estará en condiciones de generar estrategias de mercado para alcanzar dicho fin.

Así mismo se desprende nuevamente, el importante rol que viene a jugar el estudiante universitario con conocimientos de mercadotecnia, para contribuir con el migrante y permitirle tomar decisiones acertadas respecto de la mejor manera de invertir y la posibilidad de generar investigaciones de mercado que le den certeza a dichas inversiones.

Según (GHOSH, 2000), la migración de retorno es un capítulo todavía no escrito de la historia de la migración. El retorno, pone en cuestión el pretendido carácter unidireccional o definitivo de la migración, por ello debe ser explicado y tomado en cuenta a la hora de legislar sobre políticas migratorias (Durand J. , 2006).

c) Método

La presente investigación es de corte cuantitativo, de tipo transaccional o transversal, puesto que utiliza "la recopilación de datos en un momento único" (Hernández Sampieri F. C., 2010).

El nivel investigativo es descriptivo, puesto que consiste en "ubicar en una o diversas variables a un grupo de personas, 0 seres vivos, objetos, situaciones, contextos, fenómenos comunidades y así proporcionar su descripción (Hernández Sampieri F. C., 2010).

A fin de tener elementos para fundamentar las propuestas que resultaron del presente se recabó y seleccionó la información mediante la base de datos EMIF norte devueltos (2016), en relación a características tales como: género, edad, escolaridad, capacidades de trabajo, nivel de inglés y empleo entre otras, donde se manipularon deliberadamente variables ya descritas, para examinarlas con posterioridad.

El estudio se circunscribió a migrantes que durante el periodo de enero a septiembre de 2016, fueron deportados a México, cuyo número ascendió a 4,286 personas, de acuerdo a la encuesta sobre la migración en la frontera norte y sur de México (El Colegio de la Frontera Norte, 2016).

El cálculo de los principales estadísticos descriptivos, fue utilizando el paquete SPSS versión 21, haciendo cruce de variables con el procedimiento de tablas cruzadas, para encontrar información relevante de acuerdo a los resultados sobresalientes, destacando la contrastación entre la edad codificada en rangos con la escolaridad y el nivel de inglés que poseen. 


\section{HAYAZGOS:}

Para apreciar los resultados obtenidos se presenta la tabla número 1. que: de los que pretenden quedarse en México, $44.8 \%$ no tienen intención de trabajar.

Al realizar el cruce de datos de la escolaridad con relación al dominio del inglés de la muestra ya establecida, se obtuvo la siguiente información (ver Tabla 1).

Tabla $\mathbf{N}^{\circ} 1$. Resultados de la encuesta del migrante de retorno

\begin{tabular}{|c|c|c|c|}
\hline Característica & \multicolumn{3}{|c|}{ Datos } \\
\hline Género & \multicolumn{3}{|l|}{$88.8 \%$ hombres y $11.2 \%$ mujeres } \\
\hline Edad & \multicolumn{3}{|l|}{ Entre 15 y 74 años con mediana de 29 años } \\
\hline \multirow[t]{4}{*}{ Edad en rangos } & Todos & Mujeres & Hombres \\
\hline & Entre 15 y 19 años & $9.3 \%$ & $8.7 \%$ \\
\hline & Entre 20 y 39 años & $75.3 \%$ & $71.6 \%$ \\
\hline & 40 y más & $15.4 \%$ & $19.7 \%$ \\
\hline Escolaridad & \multicolumn{3}{|c|}{$\begin{array}{l}\text { Último año aprobado en México } 92.7 \% \text {; en Estados Unidos } 7.3 \% \text {, de los cuales: } 58 \% 3^{\circ} \\
\text { secundaria, } 7 \% \text { High school, }\end{array}$} \\
\hline Dominio del inglés & $\begin{array}{l}27.7 \% \text { tiene dominio del inglés, } 72.3 \% \text { no. } \\
\text { De los que hablan inglés: } \\
3.1 \% \text { habla muy bien, } 5.8 \% \text { bien y } 13.7 \% \text { regular }\end{array}$ & & \\
\hline Estado civil & \multicolumn{3}{|l|}{ Casados $32.2 \%$ Solteros $41.1 \%$} \\
\hline Oficio & \multicolumn{3}{|c|}{$\begin{array}{l}\text { Actividad agrícola } 13.6 \% \text {, Construcción } 4.7 \% \text { y conductores } 2.4 \% \text { de los cuales el } 6.6 \% \text { tomó } \\
\text { cursos de capacitación }\end{array}$} \\
\hline $\begin{array}{l}\text { Retorno al país que lo } \\
\text { deportó }\end{array}$ & \multicolumn{3}{|c|}{$\begin{array}{l}13 \% \text { piensa regresar en siete días, } 5.2 \% \text { en los primeros tres meses, } 11.9 \% \text { no sabe cuándo pero } \\
\text { piensa hacerlo. El resto no regresará. }\end{array}$} \\
\hline Posibilidades de trabajo & \multicolumn{3}{|c|}{$22 \%$ no sabe, $8.8 \%$ en la construcción, $7.1 \%$ en el campo y $44.8 \%$ no trabajará } \\
\hline Capacitación en México & \multicolumn{3}{|l|}{$3.3 \%$ si $\quad 79 \%$ no } \\
\hline Apoyo al migrante en su & \multicolumn{3}{|l|}{$12.3 \%$ se queda si recibe apoyo, el $10.5 \%$ no. } \\
\hline país origen & \multicolumn{3}{|c|}{$10.7 \%$ se habría quedado de haber recibido apoyo social. } \\
\hline
\end{tabular}

Fuente: Elaboración propia con datos de El Colegio de la Frontera Norte, (20016).

Del total de migrantes, la mayoría son del género masculino, ya que asciende al $75.3 \%$; respecto de las edades oscilan entre 20 y 39 años, resaltando que el rango entre 15 y 19 años es bajo.

Es importante resaltar que $93 \%$ tienen un grado de escolaridad de secundaria; $82 \%$ sabe escribir en español, mientras que el $0.7 \%$ solo sabe escribir en inglés, por tal, sólo el $28 \%$ tiene dominio de dicho idioma, de los cuales $3.1 \%$ lo habla muy bien, siendo grave que aun habiendo vivido por años $77.4 \%$ no tiene dominio del idioma y $60.9 \%$ no trabajó. $70 \%$ no piensa volver a Estados Unidos, mientras que $30 \%$ sí; llamando poderosamente la atención 
Tabla 2. Información cruzada de escolaridad respecto del dominio idioma inglés

\begin{tabular}{lcc}
\hline \multicolumn{1}{c}{ Edad-Escolaridad-Idioma } & Hombres & Mujeres \\
\hline Edad de 15 a 19 años, escolaridad alta, habla muy bien el inglés & 0 & 0 \\
\hline Edad de 20 a 39 años, escolaridad alta, habla muy bien el inglés & 9 & 1 \\
Edad de 40 y más, escolaridad alta, habla muy bien el inglés & 2 & 0 \\
\hline Edad de 15 a 19 años, escolaridad media, habla bien el inglés & 4 & 2 \\
Edad de 20 a 39 años, escolaridad media, habla bien el inglés & 82 & 5 \\
Edad de 40 y más, escolaridad media, habla bien el inglés & 22 & 1 \\
Edad de 15 a 19 años, escolaridad baja, habla muy bien el inglés & 1 & 0 \\
\hline Edad de 20 a 39 años, escolaridad media, habla muy bien el inglés & 0 & 0 \\
Edad de 20 a 39 años, escolaridad baja, habla muy bien el inglés & 4 & 0 \\
\hline Edad de 40 y más, escolaridad baja, habla muy bien el inglés & 1 & 0 \\
Edad de 15 a 19 años, escolaridad alta, habla bien el inglés & 0 & 0 \\
Edad de 20 a 39 años, escolaridad alta, habla bien el ingles & 0 & 0 \\
Edad de 40 y más, escolaridad media, habla regular el inglés & 88 & 8 \\
\hline Edad de 15 a 19, escolaridad alta, habla regular el inglés & 0 & 0 \\
\hline Edad de 15 a 19, escolaridad media, habla bien el inglés & 0 & 0 \\
\hline Edad de 20 a 39, escolaridad media, habla bien el inglés & 0 & 0 \\
\hline Edad de 15 a 19, escolaridad media, habla regular el inglés & 8 & 1 \\
\hline Edad de 20 a 39, escolaridad baja, habla bien el inglés & 22 & 2 \\
\hline Edad de 20 a 39, escolaridad alta, habla regular el inglés & 8 & 1 \\
\hline Edad de 20 a 39, escolaridad media, habla bien el inglés & 129 & 13 \\
\hline
\end{tabular}

Fuente: Elaboración propia con datos de tabla 1 (El Colegio de la Frontera Norte, 2016).

La población de los migrantes mexicanos en Estados Unidos, se ha mantenido constante en los últimos años, de acuerdo con la Encuesta de la Comunidad Americana (ACS). En 2015 residían en ese país 11.9 millones de mexicanos. Cifra a la que se le agregan los descendientes de la segunda y tercera generación, haciendo un total de alrededor de 35 millones de connacionales (Matías, 2017). Se estima que el contexto repercutirá en la historia migratoria entre México y Estados Unidos.

Se estima que entre 2010 y 2015 regresaron 495 mil migrantes a México, principalmente a regiones del occidente, centro y norte del país.
El Anuario Migración 2017 indica que en los siguientes años se prevé que continúe la política migratoria de Estados Unidos hacia un mayor control de la migración documentada y mayor presión sobre la indocumentada.

El retorno es una de las facetas de la migración que ha cobrado una relevancia creciente en México por lo menos desde la crisis financiera mundial de 2008. Los eventos de retorno se pueden clasificar en dos grandes categorías: forzado y voluntario. Aunque sería deseable analizar y contrastar las características entre ambas poblaciones, en la mayoría de las ocasiones la disponibilidad de información no permite segmentar a las poblaciones; en otros casos cuando se cuenta con información, el temor a la discriminación y "sentimiento de frustración" pueden influir a que las personas oculten su condición de deportado (Población, 2018). 


\section{CONCLUSIONES:}

La economía mexicana no tiene capacidad para absorber grandes masas de migrantes de retorno, incluso carece de la estabilidad económica, social y hasta política. Por lo tanto, con la información consultada se está en condiciones de decir que: el migrante que retorna a México, encuentra pocas o nulas posibilidades de desarrollo, excepto los que cuentan con patrimonio, lo invierten o ahorran.

Sin lugar a dudas, se requieren políticas públicas para apoyar al migrante que retorna, resaltando la trascendencia que tiene generar estrategias de mercado para apoyar a dicho segmento. Se requiere de programas de capacitación que le permita al migrante insertarse en el mercado laboral.

SINGER, Paul, citado por Rivera Sánchez establece que el impacto de los retornados no necesariamente es visto como uno con efectos multiplicadores de desarrollo, en la medida en que está mediado por condiciones diferenciales de los contextos. Esto es, que la relación asimétrica entre los países de origen y destino podrían imponerse al retorno y no ofrecer las condiciones suficientes para una movilidad social ascendente y quizás ni siquiera para una readaptación 0 reajuste de expectativas sociales, porque suponen que no existe un proceso de intercambio de recursos permanente entre ambos países

Por otro lado está que, tanto para salir como para retornar, se observan motivaciones económicas, que si bien es cierto que muchos de los migrantes retornados a México, desean regresar a Estados Unidos en el corto o mediano plazo, y amén de las debilidades que se aprecia tienen, es responsabilidad de su país de origen, generar condiciones para su satisfactoria reinserción social.

Siendo así que, aprovechando las capacidades adquiridas en Estados Unidos, viendo como áreas de oportunidad los avances tecnológicos y en todos los campos, se habrán de generar estrategias de mercado para que vean la posibilidad de conseguir un empleo en el mercado laboral local, aprovechar las habilidades y destrezas adquiridas en la estancia como inmigrantes que regresan a su país de origen.

Se sugiere que, eventualmente se consideren las posibilidades reales de una mejor reinserción laboral en el lugar de destino, debido a las habilidades y destrezas adquiridas en el país al que migraron, para el caso Estados Unidos, considerando el contexto institucional y social del país al que regresan, para el presente México, así como las motivaciones para tomar la decisión de regresar, en la medida de que tal acto implica una readaptación a otro ambiente, que podría conducirlo a la re-emigración, de no conseguir tal ajuste.

Partiendo de los resultados antes señalados, se está en condiciones de sugerir que, los gobiernos celebren convenios de colaboración con las instituciones de educación superior para que vía foros de participación tanto estudiantil como ciudadana, se generen políticas públicas que a partir de investigaciones de mercado realizadas por los mismos estudiantes, propongan estrategias de reinserción a dicho segmento de la población.

Es necesario considerar la apertura de nuevas fuentes de trabajo, apoyo para la creación de negocios y una capacitación adecuada para cumplir con tal fin, situación en la que de igual manera podrán integrarse estudiantes de licenciaturas afines a la administración y mercadotecnia, para con los conocimientos adquiridos, la asesoría de sus docentes y los convenios realizados con las autoridades, se logre llevar a buen puerto a los involucrados.

Por último, se reitera lo trascendente que resulta que, partiendo del presente estudio se planteen estrategias de mercado para la reinserción del migrante de retorno, garantizando así que se conviertan en personas productivas para sus lugares de residencia y no pasen a engrosar las filas del desempleo y por qué no hasta de la delincuencia.

A manera de colofón, se sugiere que los alumnos de las Unidades Académicas de formación administrativa 0 de mercadotecnia, sean quienes realicen los diagnósticos en sus estados, generen resultados y propongan estrategias de mercado para no sólo generen propuestas de integración a estudiar en sus respectivas universidades, sino que sean capaces de encontrar solución a la problemática laboral de los migrantes de retorno.

Así mismo se sugiere la celebración de convenios con las escuelas de trabajo social, para que se realicen los estudios socio-económicos requeridos, para conocer las condiciones y aspiraciones de los participantes.

De igual forma se sugiere que los estudiantes de los diferentes programas académicos conformen redes de trabajo, de esa manera podrán abordar las soluciones propuestas a la problemática señalada, de una manera integral, partiendo de las investigaciones al segmento de mercado, que proveerán de soluciones también integrales. 
Además de la capacitación y posible integración a las Universidades, han de generarse proyectos de inversión para los migrantes que retornan con recursos, o bien se les oriente sobre financiamientos atractivos.

Lo anterior se ha venido consiguiendo al generar convenios de colaboración, con las autoridades para que los estudiantes generen estudios de mercado, que planteen soluciones al desempleo mediante el autoempleo.

Respecto de la iniciativa privada, de igual manera se generan propuestas de solución vía proyectos de inversión, que inician con el correspondiente estudio de mercado.

Todo lo anterior en un marco institucional, a través de la vinculación tanto con el sector público como con el privado, a la vez pugnando por la generación de propuestas sustentables.

El estado de Zacatecas y la ahora Benemérita Universidad Autónoma de Zacatecas, ya ha implementado lo anterior y a través de los trabajos señalados se han realizado convenios con la Administración Pública y organizaciones de particulares.

Específicamente la Unidad Académica de Contaduría y Administración de la propia Universidad, tiene celebrados convenios con la Secretaría de Desarrollo Económico de Gobierno del Estado, para dar salida a los compromisos que éste tiene con la Agenda de Desarrollo Municipal, que para el presente es en la Ampliada por tratarse del aspecto Social.

De igual manera, existen convenios con Cuerpos Académicos de otras instituciones educativas, para la realización de estudios de mercado, donde los estudiantes participan en la aplicación de los correspondientes instrumentos, que para el presente han sido respecto de la situación de los migrantes.

\section{REFERENCIAS:}

Alba, F. (1999). La política migratoria mexicana después de IRCA, . e Estudios Demográaficos y Urbanos, vol. 14, núm. 1.

Alba, F. (2016). El estado de la migración. Las policas públicas ante los de la. Electrónico.

Autler, L. (1997). Una potencial alianza para el desarrollo: remesas y movimiento cooperativo en El
Salvador. Migración internacional y desarrollo. El Salvador: Fundación Nacional para el Desarrollo.

Ayers. J. W., H. R. (2009). Is Immigration a Racial Issue? Anglo Actitudes on Immigration Polices in a Border Country. . Social Science Quartely, 90(3), 593-610.

Canales, A. I. (2012). La migración mexicana frente a la crisis económica actual. Crónica de un retorno moderado. Redalyc, Ed. REMHU Revista Interdisciplinar de Mobilidade Humana, 20, 39, 116-134.

Castles \& Miller, S. M. (2004). La era de la migración. Movimientos internacionales de población en el mundo entero. Traducción: Luis Rodolfo Morán Quiroz. México: Universidad Autónoma de Zacatecas, Secretaría de Gobernación Instituto Nacional de Migración, Fundación Colosio y Miguel Ángel Posrrúa.

Delgado, W. R. (27 de Marzo de 2016). Red Internacional de Migración y Desarrollo. Obtenido de Migración, políticas públicas y desarrollo: reflexiones en torno al caso de México: https://www.researchgate.net/publication/299446 430_Migracion_politicas_publicas_y_desarrollo_r eflexiones_en_torno_al_caso_de_Mexico

Durand, J. (2006). Los inmigrantes también emigran: la migración de retorno como corolario del proceso. Revista Interdisciplinar da Mobilidade Humana, vol. 14, núm. 26-27, 167-189.

Durand, J. y. (2003). Clandestinos, migración México. Estados Unidos en los albores del siglo XXI. México.: Miguel Ángel Porrúa.

GHOSH, B. (. (2000). Return Migration: journey of hope or despair?. Geneva: International Organization of Migration.

Gómez, F. I. (2014). Derechos Humanos y Políticas Públicas. Manual. Obtenido de Las migraciones como objeto de interés de las políticas públicas: un enfoque de derechos humanos: https://core.ac.uk/download/pdf/29407234.pdf 
INEGI. (2016). Migración. Obtenido de Población-

migración. Obtenido de

http://cuentame.inegi.org.mx/poblacion/migracion.

aspx?tema=P

Matías, J. B. (2017). Anuario de migración y Remesas.

México: D. R. Fundación BBVA Bancomer,

Asociación Civil.

Mercado Vargas, H. y. (2006). La importancia Financiera,

Socioeconómica, Política y Jurídica de los

Migrantes Mexicanos. Morelia, Michoacán:

Ediciones Michoacanas.

Moctezuma L., M. (2013). Retorno de migrantes a México.

Su reformulación conceptual. Papeles de

Población, vol. 19, núm. 77, julio-septiembre, 149-175.

Moctezuma Longoria, M. (2011). Enstusiasmo estatal por la inversión productiva de los mexicanos que residen en el extranjero. Zacatecas, México: $U$. A. Zacatecas, Ed. Miguel Ángel Porrúa.

Población, C. N. (2018). Anuario de migración y remesas México 2018. México: Secretaría General del Consejo Nacional de Población.

Soria, J. P. (2017). Migrantes mexicanos en los Estados Unidos: Una revisión de la literatura sobre integración, segregación y discriminación. Esstudios Fronterizos, 18(37), 1-17. 